\title{
Analysis of the Intraventricular Electrogram for Differentiation of Distinct Monomorphic Ventricular Arrhythmias
}

\author{
SHELLY A. STEVENSON, JANICE M. JENKINS, AND LORENZO A. DiCARLO \\ From the University of Michigan, and Michigan Heart and Vascular Institute, Ann Arbor, Michigan
}

\begin{abstract}
STEVENSON, S.A., ET AL.: Analysis of the Intraventricular Electrogram for Differentiation of Distinct Monomorphic Ventricular Arrhythmias. This study investigated the effectiveness of correlation waveform analysis for identifying different ventricular electrogram morphologies of multiple VTs in the same patient. Patients with implantable antitachycardia devices are commonly subject to the occurrence of more than one distinct monomorphic VT. Each of these VTs may have unique therapeutic alternatives for termination. VTs with identical and different monomorphic configurations were recorded (1-500 Hz) using distal bipolar $(1 \mathrm{~cm})$ and distal unipolar electrograms from the right ventricular apex. Thirty-six distinct monomorphic VTs induced in 15 patients were analyzed. Nine VTs with identical morphologies (12/12 surface ECGs) were induced twice and used as a control. A template was created for each VT induced. Correlation waveform analysis was used to compare each depolarization of all other VTs induced subsequently in the same patient. The mean correlation coefficient $\left(\rho_{\mu}\right)$ of cycle-by-cycle analysis was used as a discriminant function: $\rho_{\mu} \geq 0.95$ was considered matched; and $\rho_{\mu}<0.95$ was considered distinct. From the control population, VTs were successfully classified as identical in 9 of 9 cases (100\%) using both bipolar and unipolar electrograms. VTs with different monomorphic configurations were successfully classified as being different in 31 of 33 cases (94\%) using bipolar electrogram analysis and in 29 of 33 cases (88\%) using the unipolar. Template matching is effective for detecting: (1) the recurrence of VTs, which are identical; and (2) the occurrence of a VT with a different configuration. This method appears effective using either unipolar or bipolar intracardiac waveforms. (PACE 1997; 20:2730-2738)
\end{abstract}

ventricular tachycardia, antitachycardia devices, cardioverter defibrillators, electrogram morphology, tiered therapy

\section{Introduction}

In the past decade, implantable antitachycardia devices have emerged as an alternate method of treatment for malignant cardiac tachyarrhythmias unresponsive to antiarrhythmic drugs. ${ }^{1-9} \mathrm{~Pa}$ tients who are candidates for antitachycardia devices (ATDs) are commonly subject to having more than one distinct monomorphic ventricular tachycardia (VT). In some patients, the rates of

This work was partially supported by National Science Foundation grant GER-9023514, a National Science Foundation Fellowship, and an equipment grant from Intel Corp.

Address for reprints: Janice M. Jenkins, Ph.D. Medical Computing Laboratory, Department of Electrical Engineering and Computer Science, 4421 EECS Bldg., Ann Arbor, MI 481092122. Fax: (313) 763-8041.

Received November 6, 1995; revision February 6, 1995; accepted June 20, 1996. two or more distinct VTs may be similar. However, despite similar rates, one VT may be hemodynamically well tolerated and pace terminable, while another VT may be hemodynamically destabilizing and require immediate low or high energy cardioversion. ${ }^{10-21}$ In such cases, it would be beneficial to recognize different VTs and to deliver the most effective treatment for each arrhythmia.

Several arrhythmia recognition algorithms using intracardiac electrogram morphology have been proposed for refining arrhythmia analysis. Investigated methods have focused most commonly upon separating sinus rhythm from monomorphic VT and ventricular fibrillation. ${ }^{3,4,10,22-28}$ Rate continues to be the basic classifier for discrimination of these rhythms. Morphological techniques have been advanced as a supplement to rate criteria, and given the capability of some third-generation devices to digitize 
and store the intraventricular signal, these morphometrics may play an increasing role in tachycardia identification. ${ }^{10,29,30}$ One such method, correlation waveform analysis (CWA), has been used for over two decades in coronary care monitoring and long-term ambulatory monitoring for recognition of abnormal cardiac conduction from surface electrocardiographic leads. Correlation waveform analysis is a matched filter (template based) method for discerning a change in bioelectric waveform configuration. It has been applied in recent years to intracardiac electrogram analysis and has been demonstrated to differentiate normal from abnormal cardiac rhythms. It has been shown to be effective in successfully distinguishing sinus rhythm from VT and ventricular fibrillation using intraventricular signals. ${ }^{4}$

As a follow-up to a pilot study undertaken to investigate the effectiveness of correlation waveform analysis in identifying recurrence of similar VT morphologies in the same patient, ${ }^{31}$ this study investigated the effectiveness of correlation waveform analysis in identifying different morphologies of VT in the same patient by use of VT template-matching classification. The pattern created by a particular VT was stored for subsequent recognition in a manner similar to Holter matching of "families" of ventricular premature depolarizations.

\section{Methods}

\section{Electrophysiology Study}

Recordings of ventricular electrograms were obtained during elective clinical cardiac electrophysiology studies. All of the procedures were baseline studies without antiarrhythmic drug therapy. VT was defined as having an identifiable and consistent monomorphic surface ECG QRS configuration and axis. Patients gave informed written consent and were studied subsequently in a fasting postabsorbtive state after sedation with 1-3 mg of intraveneous medazalom. After administering $1 \%$ lidocaine for local anesthesia, one 7 French and two 6 French side-arm sheaths (Cordis Corporation, Miami, FL, USA) were positioned in the right femoral vein using the Seldinger technique. Three 6 French quadripolar electrode catheters with an interelectrode distance of $1 \mathrm{~cm}$ (USCI division, C.R. Bard Inc., Billerica, MA,
USA) were introduced and advanced under fluoroscopic guidance. Each electrode catheter had four platinum electrodes with an interelectrode space of $1 \mathrm{~cm}$. A platinum electrode at the tip of each catheter consisted of a half-sphere $2 \mathrm{~mm}$ in diameter attached to a cylinder $2 \mathrm{~mm}$ in diameter and $1 \mathrm{~mm}$ in length. Proximal to the tip electrode were three platinum electrode rings that were 2 $\mathrm{mm}$ in diameter and $2 \mathrm{~mm}$ in length. The resulting surface area of the distal pair of electrodes was $12.6 \mathrm{~mm}^{2}$. One electrode catheter was positioned in the high right atrium or right atrial appendage. Two electrode catheters were positioned in the right ventricular apex for right ventricular apical pacing and recording, respectively. Electrograms were recorded on FM magnetic tape $(9.525 \mathrm{~cm} / \mathrm{s})$ from distal bipolar and unipolar endocardial electrodes positioned in the right ventricular apex. Unipolar configurations were recorded from the distal member of the right ventricular apex catheter with a reference guidewire located at the femoral site of catheter insertion. Bipolar configurations were recorded from the distal pair of electrodes of the right ventricular apex catheter. Amplifier settings were $1-500 \mathrm{~Hz}$ and gains were held constant throughout the recordings. Filter settings of $1-500 \mathrm{~Hz}$ were chosen to obtain a wideband intracardiac electrogram for morphological analysis. ${ }^{4}$ Electrograms were digitized at $1,000 \mathrm{~Hz}$ and processed offline by custom software on an Intel 80486DX microcomputer.

\section{Patient Population}

The patient population consisted of 16 men and 2 women (age range 45-79). This study was performed on consecutive patient recordings over a 6-month period who met the inclusion criterion of multiple inductions of one or more morphologically distinct VTs. ${ }^{32}$ All patients had coronary artery disease with history of myocardial infarction. In eight patients, two separate inductions of a VT identical in 12 of 12 standard surface ECGs were compared and used as a control. In 1 of these 8 patients, a second monomorphic VT was also induced twice and used as a control. Thus, nine paired sets of induced identical monomorphic VTs were available for analysis as a control sample.

Five of the eight patients with control (identical) VTs and ten additional patients evaluated 
in this study had two or more monomorphic VTs with distinct configurations. Twelve of these 15 patients (80\%) had two distinct VT morphologies, 2 patients (13\%) had three distinct VT morphologies and 1 patient $(7 \%)$ had six distinct VT mor- phologies. Consecutive inductions of each VT with a different monomorphic configuration were labeled using the convention, $\mathrm{VT}_{1}, \mathrm{VT}_{2}$, etc. (Table I). In cases with more than two VT configurations in the same patient, $\mathrm{VT}_{1}$ was compared

Table I.

Description of distinct Ventricular Tachycardias

\begin{tabular}{|c|c|c|c|c|c|c|}
\hline Patient & BBB & Axis & Plane & $\mathrm{CL}(\mathrm{ms})$ & VT \# & Termination \\
\hline \multirow[t]{2}{*}{ AAEL204 } & RBBB & $\mathrm{S}$ & $\mathrm{R}$ & 330 & $\mathrm{VT}_{1}$ & $P$ \\
\hline & RBBB & S & $\mathrm{R}$ & 370 & $\mathrm{VT}_{2}$ & $P$ \\
\hline \multirow[t]{2}{*}{ AAEL206 } & RBBB & S & $\mathrm{L}$ & 370 & $\mathrm{VT}_{1}$ & $\mathrm{Sp}$ \\
\hline & RBBB & S & $\mathrm{L}$ & 370 & $\mathrm{VT}_{2}$ & $\mathrm{P}$ \\
\hline \multirow[t]{3}{*}{ AAEL212 } & LBBB & 1 & $\mathrm{R}$ & 420 & $\mathrm{VT}_{1}$ & $P$ \\
\hline & LBBB & I & $\mathrm{R}$ & 420 & $\mathrm{VT}_{2}$ & C \\
\hline & LBBB & 1 & $\mathrm{R}$ & 420 & $\mathrm{VT}_{3}$ & C \\
\hline \multirow[t]{2}{*}{ AAEL216 } & LBBB & S & $\mathrm{L}$ & 290 & $\mathrm{VT}_{1}$ & In \\
\hline & RBBB & S & $\mathrm{R}$ & 230 & $\mathrm{VT}_{2}$ & C \\
\hline \multirow[t]{2}{*}{ AAEL220 } & RBBB & S & $\mathrm{R}$ & 450 & $\mathrm{VT}_{1}$ & Sp \\
\hline & RBBB & S & L & 310 & $\mathrm{VT}_{2}$ & C \\
\hline \multirow[t]{2}{*}{ AAEL228 } & LBBB & S & $\mathrm{L}$ & 300 & $\mathrm{VT}_{1}$ & C \\
\hline & RBBB & S & $\mathrm{R}$ & 290 & $\mathrm{VT}_{2}$ & C \\
\hline \multirow[t]{3}{*}{ AAEL233 } & RBBB & S & $\mathrm{L}$ & 290 & $\mathrm{VT}_{1}$ & $P$ \\
\hline & RBBB & S & $\mathrm{R}$ & 320 & $\mathrm{VT}_{2}$ & In \\
\hline & RBBB & 1 & $\mathrm{R}$ & 270 & $\mathrm{VT}_{3}$ & C \\
\hline \multirow[t]{2}{*}{ AAEL236 } & LBBB & 1 & $\mathrm{~L}$ & 220 & $\mathrm{VT}_{1}$ & C \\
\hline & LBBB & 1 & $\mathrm{R}$ & 180 & $\mathrm{VT}_{2}$ & C \\
\hline \multirow[t]{2}{*}{ AAEL241 } & RBBB & $S$ & $\mathrm{~L}$ & 270 & $\mathrm{VT}_{1}$ & C \\
\hline & RBBB & $S$ & $\mathrm{R}$ & 230 & $\mathrm{VT}_{2}$ & C \\
\hline \multirow[t]{2}{*}{ AAEL253 } & LBBB & $\mathrm{S}$ & $\mathrm{R}$ & 220 & $\mathrm{VT}_{1}$ & C \\
\hline & RBBB & 1 & $\mathrm{R}$ & 220 & $\mathrm{VT}_{2}$ & $P$ \\
\hline \multirow[t]{2}{*}{ AAEL265 } & RBBB & $S$ & $\mathrm{R}$ & 470 & $\mathrm{VT}_{1}$ & Sp \\
\hline & RBBB & $S$ & $\mathrm{R}$ & 470 & $\mathrm{VT}_{2}$ & Sp \\
\hline \multirow[t]{6}{*}{ AAEL266 } & LBBB & $\mathrm{S}$ & $\mathrm{L}$ & 470 & $\mathrm{VT}_{1}$ & In \\
\hline & RBBB & S & L & 450 & $\mathrm{VT}_{2}$ & In \\
\hline & LBBB & S & $L$ & 450 & $\mathrm{VT}_{3}$ & In \\
\hline & RBBB & $S$ & $\mathrm{R}$ & 470 & $\mathrm{VT}_{4}$ & In \\
\hline & RBBB & $\mathrm{S}$ & $L$ & 450 & $\mathrm{VT}_{5}$ & In \\
\hline & LBBB & S & L & 470 & $\mathrm{VT}_{6}$ & C \\
\hline \multirow[t]{2}{*}{ AAEL280 } & RBBB & 1 & $\mathrm{R}$ & 310 & $\mathrm{VT}_{1}$ & Sp \\
\hline & LBBB & S & $\mathrm{L}$ & 250 & $\mathrm{VT}_{2}$ & C \\
\hline \multirow[t]{2}{*}{ AAEL296 } & RBBB & 1 & $\mathrm{R}$ & 330 & $\mathrm{VT}_{1}$ & $P$ \\
\hline & RBBB & 1 & $\mathrm{R}$ & 270 & $\mathrm{VT}_{2}$ & $P$ \\
\hline \multirow[t]{2}{*}{ AAEL308 } & RBBB & I & L & 250 & $\mathrm{VT}_{1}$ & $P$ \\
\hline & RBBB & $\mathrm{S}$ & L & 270 & $\mathrm{VT}_{2}$ & $P$ \\
\hline
\end{tabular}

AAELxxx = patient number; Ann Arbor Electrogram Libraries, Ann Arbor, MI.

$\mathrm{BBB}=$ bundle branch block; $\mathrm{C}=$ cardioversion; $\mathrm{CL}=$ cycle length; $\mathrm{In}=$ induced different $\mathrm{VT} ; \mathrm{I}=$ inferior; $\mathrm{L}$ $=$ left $\mathrm{ms}=$ milliseconds; $\mathrm{P}=$ antitachycardia pacing, $\mathrm{R}=$ right; $\mathrm{S}=$ superior; $\mathrm{Sp}=$ spontaneous. 
to $\mathrm{VT}_{2}, \mathrm{VT}_{2}$ to $\mathrm{VT}_{3}$, and $\mathrm{VT}_{1}$ to $\mathrm{VT}_{3}$, etc. such that all possible combinations in the same patient were considered. Overall, 36 different monomorphic VTs were induced and analyzed (see Table II).

\section{Method of Analysis}

Correlation waveform analysis was used to classify morphological differences in the intracardiac electrograms. ${ }^{4}$ Templates were constructed of each induced VT by signal averaging 10-15 cycles and were used for cycle-by-cycle comparison of each subsequently induced VT in the same patient. Three VTs occurred spontaneously during

Table II.

Description of Distinct Paired Sets on a Patient by Patient Basis

\begin{tabular}{|c|c|c|c|}
\hline Patient & $\begin{array}{l}\text { \# of } \\
\text { Distinct } \\
\text { VTs }\end{array}$ & $\begin{array}{l}\text { \# of } \\
\text { Paried } \\
\text { Sets }\end{array}$ & $\begin{array}{c}\text { Combinational } \\
\text { Groupings }\end{array}$ \\
\hline
\end{tabular}

\begin{tabular}{lrrc} 
AAEL204 & 2 & 1 & \\
AAEL206 & 2 & 1 & \\
AAEL212 & 3 & 3 & $\{1,2\}\{1,3\}\{2,3\}$ \\
AAEL216 & 2 & 1 & \\
AAEL220 & 2 & 1 & \\
AAEL228 & 2 & 1 & \\
AAEL233 & 3 & 3 & $\{1,2\}\{1,3\}\{2,3\}$ \\
AAEL236 & 2 & 1 & \\
AAEL241 & 2 & 1 & \\
AAEL253 & 2 & 1 & \\
AAEL265 & 2 & 1 & \\
AAEL266 & 6 & 15 & $\{1,2\}\{1,3\}\{1,4\}\{1,5\}$ \\
& & & $\{1,6\}\{2,3\}\{2,4\}$ \\
& & & $\{2,5\}\{2,6\}\{3,4\}$ \\
AAEL280 & 2 & 1 & $\{3,5\}\{3,6\}\{4,5\}$ \\
AAEL296 & 2 & 1 & $\{4,6\}\{5,6\}$ \\
AAEL308 & 2 & 1 & \\
15 & 36 & 33 & \\
\hline
\end{tabular}

In this study, there were 36 distinct ventricular tachycardia morphologies within 15 patients. Overall, thirty-three paired sets of distinct ventricular tachycardia configurations were investigated. Numbers seen in the right-most column indicate the tachycardia combination being tested, i.e., $\{1,2\}$ is the combination of $\mathrm{VT}_{1}$ with $\mathrm{VT}_{2}$.

AAELxxx = patient number, Ann Arbor Electrogram Libraries, Ann Arbor, MI. electrophysiology studies. The remainder were induced by programmed stimulation. Cycles used to construct the template were taken from stable monomorphic VT. For example, a $\mathrm{VT}_{1}$ template was tested against each individual $\mathrm{VT}_{2}$ cycle and a $\mathrm{VT}_{2}$ template was constructed and tested against each $\mathrm{VT}_{1}$ cycle. This procedure was repeated for all possible combinations.

The value of the correlation coefficient, $\rho$, is a measure of the similarity of two waveforms, i.e., the template versus a depolarization under analysis, with a value of 1 indicating a perfect match. The correlation coefficient, $\rho$, is given by:

$$
\rho=\frac{\sum_{i=1}^{N}(T i-T)(S i-S)}{\sqrt{\sum_{i=1}^{N}(T i-T)^{2} \sum_{i=1}^{N}(S i-S)^{2}}}
$$

where $\mathrm{N}=$ number of template points, $\mathrm{T}_{\mathrm{i}}=$ template points, $\mathrm{S}_{\mathrm{i}}=$ signal points under analysis, $\mathrm{T}$ = average of the template points, and $\mathrm{S}=$ average of the signal points under analysis. The normalized correlation is independent of amplitude and baseline fluctuations and only measures relative shape differences between the two signals.

A software trigger was used to align the template with the cycle being tested. Eleven values of $\rho$ were computed for each waveform. First the waveform was aligned at the original trigger location and the calculation was repeated by shifting in a stepwise fashion 5 points to the left and 5 points to the right of the original alignment. The maximum value of these 11 correlations reflected optimal alignment and was taken to be the true correlation coefficient for that depolarization. ${ }^{28,31,33}$ Mean correlation coefficients $\left(\rho_{\mu}\right)$ for each VT passage were calculated separately for (1) the bipolar lead configuration; and (2) the unipolar lead configuration. If $\rho_{\mu}$ was greater than or equal to the threshold value of 0.95 when the ith VT template was compared to all the cycles of the $j$ th VT and when the $j$ th VT template was compared to all the cycles of the ith VT (bilateral comparison), then the two VTs were classified as identical or matched, where $i$ and $j$ are indices of successive VT induction in the same patient. If $\rho_{\mu}$ was $<0.95$ in a bilateral comparison of the two VTs, they were classified as distinct or unmatched. 


\section{Results}

Quantitative results of the nine paired control sets (reinduction of VT with identical morphology) are given in Table III, and quantitative results of the 33 paired sets of different morphologies are given in Table IV. In the control population, the mean correlation coefficient consistently equaled or exceeded the threshold value of 0.95 , indicating that the second induction of an identical VT was successfully found to be the same in 9 of $9(100 \%)$ cases in both unipolar and bipolar recordings.

Of the 33 paired sets of 36 distinct VTs having differing morphologies, the mean correlation coefficient $(\rho \mu)$ of the bipolar analysis fell below the threshold value of 0.95 , successfully classifying VTs as distinct, in 31 of 33 (94\%) cases. In 29 of 33 $(88 \%)$ cases using unipolar analysis, different VTs were classified as distinct. Absolute failure to detect distinct VTs using both unipolar and bipolar electrograms occurred in 2 of $33(6 \%)$ cases (AAEL220 and AAEL266H). Four randomly selected patients, one from the control population and three with more than one distinct VT, were reanalyzed to confirm the reproducibility of each correlation. Mean correlation coefficients of re-

Table III.

Identical Ventricular Tachycardias

\begin{tabular}{ccc}
\hline \multicolumn{1}{c}{ Patient } & Bipolar & Unipolar \\
\hline AAEL204 VT & 0.995 & 0.994 \\
AAEL206 VT & 0.972 & 0.994 \\
AAEL211 VT & 0.988 & 0.987 \\
AAEL239 VT & 0.998 & 0.999 \\
AAEL263 VT & 0.981 & 0.986 \\
AAEL265 VT & 0.992 & 0.994 \\
AAEL266 VT & 0.991 & 0.995 \\
AAEL308A VT & 0.996 & 0.972 \\
AAEL308B VT & 0.997 & 0.989 \\
\hline
\end{tabular}

Quantitative results of the nine paired control sets (reinduction of ventricular tachycardia with identical morphology). A mean correlation coefficient $\geq 0.95$ is defined as successful matching of two inductions of the same VT. In the control population, the mean correlation coefficient consistently equaled or exceeded the threshold value of 0.95 , indicating that the second induction of an identical ventricular tachycardia was successfully found to be the same in $9 / 9(100 \%)$ cases in both unipolar and bipolar recordings. AAELxxx = Patient number, Ann Arbor Electrogram Libraries, Ann Arbor, MI.
Table IV.

Distinct Ventricular Tachycardias

\begin{tabular}{|c|c|c|}
\hline Patient & Bipolar & Unipolar \\
\hline AAEL204 VT & 0.206 & 0.172 \\
\hline AAEL206 VT & 0.731 & 0.928 \\
\hline AAEL212A VT & 0.378 & 0.909 \\
\hline AAEL212B VT $1 / 3$ & 0.514 & 0.942 \\
\hline AAEL212C VT $2 / 3$ & 0.945 & 0.991 \\
\hline AAEL216 $\mathrm{VT}_{1 / 2}$ & 0.246 & 0.937 \\
\hline AAEL220 VT & 0.977 & 0.991 \\
\hline AAEL228 $\mathrm{VT}_{1 / 2}$ & 0.637 & -0.218 \\
\hline AAEL233A VT & 0.583 & 0.303 \\
\hline AAEL233B VT $1 / 3$ & 0.217 & 0.361 \\
\hline AAEL233C VT $2 / 3$ & 0.920 & 0.789 \\
\hline AAEL236 $\mathrm{VT}_{1 / 2}$ & 0.776 & 0.863 \\
\hline AAEL241 VT $1 / 2$ & 0.906 & 0.386 \\
\hline AAEL253 $\mathrm{VT}_{1 / 2}$ & 0.715 & 0.900 \\
\hline AAEL265 VT $1 / 2$ & -0.336 & 0.902 \\
\hline AAEL266A VT & 0.171 & 0.434 \\
\hline AAEL266B VT $1 / 3$ & 0.798 & 0.552 \\
\hline AAEL266C VT ${ }_{1 / 4}$ & 0.711 & 0.136 \\
\hline AAEL266D VT ${ }_{1 / 5}$ & 0.324 & 0.465 \\
\hline AAEL266E VT ${ }_{1 / 6}$ & 0.126 & 0.347 \\
\hline AAEL266F VT $2 / 3$ & -0.443 & 0.907 \\
\hline AAEL266G VT $2 / 4$ & 0.309 & 0.612 \\
\hline AAEL266H VT $2 / 5$ & 0.975 & 0.997 \\
\hline AAEL266I VT & 0.421 & 0.962 \\
\hline AAEL266J VT & 0.309 & 0.658 \\
\hline AAEL266K VT & -0.396 & 0.931 \\
\hline AAEL266L VT & -0.045 & 0.936 \\
\hline AAEL266M VT $_{4 / 5}$ & 0.652 & 0.626 \\
\hline AAEL266N VT & 0.171 & 0.829 \\
\hline AAEL266O VT $5 / 6$ & 0.262 & 0.94 \\
\hline AAEL280 $\mathrm{VT}_{1 / 2}$ & 0.585 & 0.460 \\
\hline AAEL296 VT & -0.335 & 0.775 \\
\hline AAEL308 VT & 0.942 & 0.759 \\
\hline
\end{tabular}

Quantitative results of the thirty-three paired sets of different ventricular tachycardia morphologies. The designation $\mathrm{VT}_{\mathrm{ij}}$ indicates that the templates of the ith and jth ventricular tachycardia morphologies for the given patient were analyzed. A mean correlation coefficient $<0.95$ is defined as a successful classification of distinct VTs. A mean correlation coefficient $\geq 0.95$ is defined as a failure to classify as different or unmatched. In bipolar analysis $31 / 33(94 \%)$ cases were successfully classified as distinct. In unipolar analysis $29 / 33(88 \%)$ cases were successfully classfied as distinct.

peated analyses were found to be similar within 0.01 . Exemplary passages of electrograms showing two inductions of identical VTs in the same patient is seen in Figure 1. Exemplary passages de- 


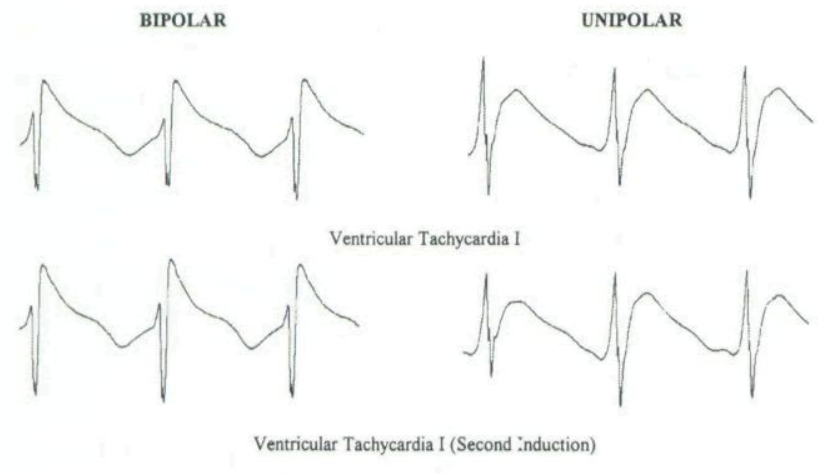

Figure 1. Two inductions of identical VTs in the same patient (AAEL204). The upper left panel shows exemplary waveforms from a bipolar ventricular electrogram of the first induction of $V T_{1}$, and the upper right panel is a unipolar electrogram of the same segment. The lower left and right panels are bipolar and unipolar electrograms from the second induction of an identical VT (confirmed by a 12-lead ECG). Mean correlation coefficients were $\geq 0.95$ in bilateral comparisons of both bipolar and unipolar configurations. The mean correlation coefficient, $\rho \mu$, was 0.995 in this patient using the bipolar electrogram and 0.994 using the unipolar electrogram.

picting inductions of two distinct VTs in the same patient are shown in Figures 2 and 3.

Eleven of the 36 distinct VTs required cardioversion for termination, 8 responded to antitachycardia pacing, 5 terminated spontaneously, and in 7 , attempts of antitachycardia pacing resulted in the induction of different VTs. The average cycle length of VTs requiring cardioversion was $290 \mathrm{~ms}$. The average cycle length of VTs responding to antitachycardia pacing was $310 \mathrm{~ms}$. The average cycle length of VTs spontaneously terminating was $440 \mathrm{~ms}$. In 8 of the 9 paired control sets, antitachycardia pacing terminated the arrhythmia. In the remaining control set, one induction of the VT spontaneously terminated, while the other induction required cardioversion. In 5 of $15(33 \%)$ patients having more than one distinct VT, more than one method of antitachycardia therapy was required for termination of all VTs. In the remainder, a single method of antitachycardia treatment terminated all VTs (Table I).

\section{Discussion}

From the results of the present study, morphometric analysis using template-matching cor- relation waveform analysis of either bipolar or unipolar intracardiac leads appears to be effective in identifying different morphologies of VT in the same patient. Recent studies have demonstrated the validity of time-domain electrogram analysis of VT,,$^{424-28}$ intraatrial electrogram analysis of retrograde conduction, ${ }^{33}$ and recognition of subsequent recurrence of an identical VT by templatematching techniques. ${ }^{31}$ Previous work has found that bipolar and unipolar electrograms were equally effective in distinguishing VT from sinus rhythm. ${ }^{22}$ In this study, successful classification of distinct VTs was similar using CWA of the bipolar electrogram and the unipolar electrogram. Using the bipolar electrogram 31 of $33(94 \%)$ were classified correctly while 29 of $33(88 \%)$ were classified correctly using the unipolar electrogram. Thus pattern recognition technique of morphological classification may provide a means for confirming the presence of the induced or documented VTs for which therapy has been prescribed recognizing the emergence of a new VT. However, it should be noted that in 4 of $33(12 \%)$ cases of bipolar analysis and in 9 of $33(27 \%)$ cases of unipolar analysis the mean correlation coefficient $(\rho \mu)$ fell between 0.90 and 0.95 . The mean correlation coefficient $(\rho \mu)$ fell below 0.90 in 27 of

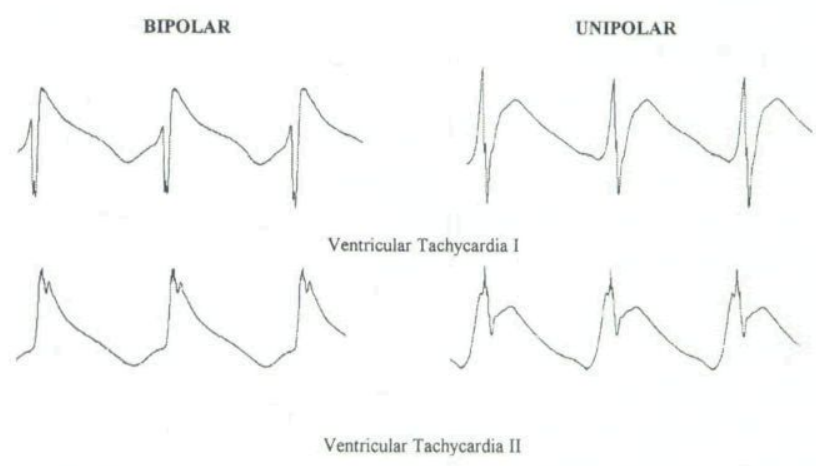

Figure 2. Inductions of two distinct monomorphic VTs in the same patient (AAEL204). The upper left panel shows exemplary waveforms from a bipolar ventricular electrogram of $V T_{1}$, and the upper right panel is a unipolar electrogram of the same segment. The lower left and right panels are bipolar and unipolar recordings respectively of $\mathrm{VT}_{2}$. Morphometric analysis classified these as distinct in both configurations. The mean correlation coefficient, $\rho \mu$, was 0.206 in this patient using the bipolar electrogram and 0.172 using the unipolar electrogram. 
BIPOLAR

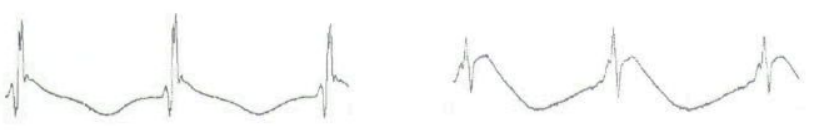

Ventricular Tachycardia I

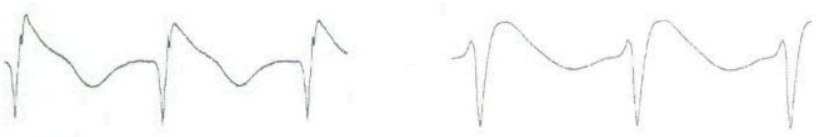

Ventricular Tachycardia II

Figure 3. Inductions of two distinct monomorphic VTs in the same patient (AAEL266). This figure is ordered as in Figure 2. Classification of distinct VTs was made in both bipolar and unipolar recordings. The mean correlation coefficient, was 0.171 in this patient using the bipolar electrogram and 0.434 using the unipolar electrogram.

$33(82 \%)$ cases of bipolar analysis and in 20 of 33 $(61 \%)$ cases of unipolar analysis. The mean correlation coefficient $(\rho \mu)$ exceeded 0.95 in 2 of 33 $(6 \%)$ cases of bipolar analysis and in 4 of $33(12 \%)$ of unipolar analysis. This suggests that the bipolar electrograms may more readily discriminate between VTs of distinct morphologies than the unipolar electrograms.

In earlier generation devices, patients with VTs have been treated with the delivery of a high energy shock, ${ }^{3,5,6,9}$ but modern antitachycardia devices offer new diagnostic and therapeutic alternatives to treat tachyarrhythmias. ${ }^{11,13,17-19}$ Tiered therapy multiprogrammable antiarrhythmia devices are capable of antitachycardia pacing, cardioversion, and defibrillation. Choices of therapeutic options permit an aggressive response to VTs, which are known to be hemodynamically destabilizing. Conversely, antitachycardia pacing may be the appropriate choice for hemodynamically stable VTs. ${ }^{9,10}$ The option of treating VTs with antitachycardia pacing eliminates the pain and trauma associated with cardioversion and avoids premature battery depletion. Comparative efficacy studies have shown that both antitachycardia pacing and low energy cardioversion are capable of terminating VTs as well as accelerating them. Different VTs respond differently to antitachycardia pacing and low energy cardioversion, and response to one therapy does not predict response to another. ${ }^{11-19}$
In the present study, the rate of the arrhythmia did not appear to determine hemodynamic stability. Slower VTs sometimes required cardioversion for termination, while some faster VTs were terminated with antitachycardia pacing (see Table I). In the control group, it was observed that the same method of treatment could be used to terminate repeated inductions of the same VT. The results of this study suggest that additional methods of classification need to be considered in addition to the rate criterion, to determine therapeutic options for treating VTs. This study demonstrates that morphological analysis may be one means of successfully differentiating multiple ventricular arrhythmias.

With the development of devices now capable of tiered therapy has come the incorporation of rate zones to allow tailored therapy for slow and fast tachyarrhythmias. ${ }^{9,10}$ The tachycardia interval (in ms) and tachycardia duration (number of depolarizations) used for tachycardia detection within each rate zone is independently programmable. To accommodate "electrogram dropout," which occurs in low amplitude ventricular fibrillation signals, the ventricular fibrillation detection zone is often set to include ranges that encompass monomorphic VT cycle lengths. While such programming is intended to prevent the misclassification of ventricular fibrillation as a less malignant VT, it imposes the use of more aggressive device therapy than may be necessary for VTs amenable to antitachycardia pacing. In one study, ${ }^{9}$ rapid monomorphic VT with cycle lengths falling within the fibrillation detection zone accounted for 72 of $120(60 \%)$ spontaneous tachyarrhythmias treated as ventricular fibrillation. In addition to discriminating monomorphic VT with differing configurations, correlation waveform analysis has also been demonstrated to distinguish monomorphic VT from ventricular fibrillation. ${ }^{27}$

Thus, incorporation of automated electrogram analysis has the potential of dramatically refining antitachycardia device therapy. The clinical importance of considering morphology of intracardiac electrograms in identifying arrhythmias is further implied from recent studies using retrieval of electrograms stored in antitachycardia devices. In one study of 16 patients, ${ }^{29}$ three patients received out-of-hospital shocks, and verifi- 
cation of the initiating event from the stored electrogram was recovered via telemetry. A clinical diagnosis was made in all three cases (atrial fibrillation, polymorphic VT, and rate sensing lead disruption, respectively) by visual inspection of the signal characteristics and morphology of the stored electrograms. In another study ${ }^{30}$ the morphology of the initiating events of monomorphic VT was visually inspected in an attempt to identify mechanisms of initiation of VT. Morphological classification was performed (presumably by subjective means, since no description is given of the method of classifying morphologies), but there was little question that waveforms of differing morphology were visible in the stored electrogram, and the morphology of the tachycardia was distinctly different from sinus rhythm. This was evident despite minimal analog-to-digital sampling rates, limited storage, and severely limited band-pass filtering, which is characteristic of battery operated antitachycardia devices.

\section{Limitations}

Although template-matching appears to be an effective method, it requires multiple calculations per waveform and poses an additional computational demand. Potential implementation of this pattern recognition process in future battery operated implantable devices would best be realized in special purpose hardware for efficiency of computation and low power consumption. The use of digital signal processing (DSP) chips for a hardware implementation of correlation has been demonstrated, in which all processing for each cycle was completed in $30 \mu \mathrm{s} .{ }^{34}$ DSP chips, although fast, are still too power consumptive at present to be considered for implantable devices, but the design of special purpose VLSI chips of low power is entirely feasible.

\section{References}

1. Olson W, Bardy G, Mehra R, et al. Onset and stability for ventricular tachycardia detection in an implantable pacer-cardioverter-defibrillator. Comp Cardiol 1987; 34:167-170.

2. Ripley KL, Bump TE, Arzbaecher RC. Evaluation of techniques for recognition of ventricular arrhythmias by implanted devices. IEEE Trans Biomed Eng 1989; 36:618-624.
Electrogram morphology sometimes varies at the onset of arrhythmias. The creation of the template would require time for monomorphic stabilization. However, the capability of ATDs to store multiple electrograms permits off-line template creation of any emerging VTs to be used for subsequent inductions. Correlation waveform analysis was used as a morphometric in this study in order to demonstrate the feasibility of morphological distinction and classification of multiple VTs. Less demanding algorithms have been developed and have been shown to be as effective as correlation waveform analysis in distinguishing VT from sinus rhythm. ${ }^{28,35}$ It remains to be shown whether these methods perform as well in classification of distinct VTs in the same patient. The present study provides encouraging results and suggestions that intraventricular electrograms should be examined further with other classifiers.

\section{Summary}

This study and others provide a growing body of evidence that human evaluation of stored electrograms can be duplicated by automated methods. Characterization of distinct VTs, using VTs induced during clinical electrophysiology studies as well as VTs stored in the memory of antitachycardic devices, appears feasible utilizing unique morphological features derived for design of subsequent recognition algorithms. The addition of morphology to the rate algorithm would allow quicker recognition of VTs known to be hemodynamically unstable, as well as identification of those known to be pace terminable. For patients with multiple VTs and/or rapid VTs, the added capability of recognizing distinct VTs would allow the selection of the most appropriate and least discomforting therapy.

3. Mirowski M, Mower MM, Reid PR. The automatic implantable defibrillator. Am Heart J 1980; 100(6II):1089-1092.

4. Lin D, DiCarlo LA, Jenkins JM. Identification of ventricular tachycardia using intracavitary ventricular electrograms: Analysis of time and frequency domain patterns. PACE 1988; 11:15921606. 
5. Watkins L, Mirowski M, Mower MM, et al. Automatic defibrillation in man. J Thorac Cardivasc Surg 1981; 82:492-500.

6. Griffin JC, Mason JW, Calfee RV. Clinical use of an implantable automatic tachycardia-terminating pacemaker. Am Heart J 1980; 100:1093-1096.

7. Tooley MA, Davies DW, Nathan AW, et al. Recognition of multiple tachyarrhythmias by rate-independent means using a small microcomputer. PACE 1991; 14:337-340.

8. Fisher JD, Goldstein M, Ostrow E, et al. Maximal rate of tachycardia development: Sinus tachycardia with sudden exercise vs. spontaneous ventricular tachycardia. PACE 1983; 6:221-228.

9. Bardy GH, Hofer B, Johnson G, et al. Implantable transvenous cardioverter-defibrillators. Circulation 1993; 87:1152-1168.

10. DiCarlo LA, Jenkins JM, Caswell SA, et al. Tachycardia detection by antitachycardia devices: Present limitations and future strategies. J Intervent Cardiol 1994; 7:459-472.

11. Bardy GH, Troutman RN, Poole JE, et al. Clinical experience with a tiered-therapy multiprogrammable antiarrhythmia device. Circulation 1992; 85:1689-1698.

12. Bardy GH, Poole JE, Kudenchuk PJ, et al. A prospective randomized repeat-crossover comparison of antitachycardia pacing with low-energy cardioversion. Circulation 1993; 87:1889-1896.

13. Fromer M, Brachman J, Block M, et al. Efficacy of automatic multimodal device therapy for ventricular tachyarrhythmias as delivered by new implantable pacing cardioverter-defibrillator. Circulation 1992; 86:363-374.

14. Saksena S, Chandran P, Shah Y, et al. Comparative efficacy of transvenous cardioversion and pacing in patients with sustained ventricular tachycardia: A prospective, randomized, crossover study. Circulation $1985 ; 72: 153-160$.

15. Charos GS, Haffajee CI, Gold RL, et al. A theoretically and practically more effective method for interruption of ventricular tachycardia: Self-adapting autodecremental overdrive pacing. Circulation 1986; 73:309-315.

16. Estes NAM III, Haugh CJ, Wang PJ, et al. Antitachycardia pacing and low-energy cardioversion for ventricular tachycardia termination: A clinical perspective. Am Heart J 1994; 127:1038-1046.

17. Fromer M, Schlapfer J, Fischer A, et al. Experience with a new implantable pacer-cardioverter-defibrillator for the therapy of recurrent sustained ventricular tachyarrhythmias: A step toward a universal ventricular tachyarrhythmia control device. PACE 1991; 14:1288-1298.

18. Leitch JW, Gillis AM, Wyse G, et al. Reduction in defibrillator shocks with an implantable device combining antitachycardia pacing and shock therapy. J Am Coll Cardiol 1991; 18:145-151.

19. Saksena S, Mehta D, Krol RB, et al. Experience with a third-generation implantable cardioverter-defibrillator. Am J Cardiol 1991; 67:1375-1384.
20. Swerdlow CD, Peng-Sheng C, Kass RM, et al. Discrimination of ventricular tachycardia from sinus tachycardia and atrial fibrillation in a tiered-therapy cardioverter-defibrillator. J Am Coll Cardiol 1994; 23:1342-1355.

21. Hamer AWF, Rubin SA, Peter T, et al. Factors that predict syncope during ventricular tachycardia in patients. Am Heart J 1984; 107:997-1005.

22. Greenhut SE, DiCarlo LA, Jenkins JM, et al. Identification of ventricular tachycardia using intracardiac electrograms: A comparison of unipolar versus bipolar waveform analysis. PACE 1990; 14:427-433.

23. Morris MM, Jenkins JM, Lorenzo LA. Band-limited morphometric analysis of the intracardiac signal: Implications for antitachycardia devices. PACE (Submitted for publication, May 1995).

24. Landberg JJ, Gibb WJ, Auslander DM, et al. Identification of ventricular tachycardia with use of morphology of the endocardial electrogram. Circulation 1988; 77:1363-1369.

25. Paul VE, O'Nunain S, Milik M, et al. Temporal electrogram analysis: Algorithm development. PACE 1990; 13(II):1943-1947.

26. Davies DW, Wainwright RJ, Tooley MA, et al. Detection of pathologic tachycardia by analysis of electrogram morphology. PACE 1986; 9:200-208.

27. DiCarlo LA, Jenkins JM, Winston SA, et al. Differentiation of ventricular tachycardia from ventricular fibrillation using intraventricular electrogram morphology. Am J Cardiol 1992; 70:820-822.

28. Throne RD, Jenkins JM, Winston SA, et al. A comparison of four new time domain methods for discrimination monomorphic ventricular tachycardia from sinus rhythm using ventricular waveform morphology. IEEE Trans Biomed Eng 1991; 38(6):561-570.

29. Hook BG, Marchlinski FE. Value of ventricular electrogram recordings in the diagnosis of arrhythmias precipitating electrical device therapy. J Am Coll Cardiol 1991; 17:985-990.

30. Roelke M, Garan H, McGovern BA, et al. Analysis of the initiation of spontaneous monomorphic ventricular tachycardia by stored intracardiac electrograms. J Am Coll Cardiol 1994; 23:117-122.

31. Throne RD, Jenkins JM, Winston SA, et al. Use of tachycardia templates for recognition of recurrent monomorphic ventricular tachycardia. Comp Cardiol 1989: 171-174.

32. Ann Arbor Electrogram Libraries, Ann Arbor, MI.

33. Throne RD, Jenkins JM, Winston SA, et al. Discrimination of retrograde from anterograde atrial activation using intracardiac electrogram waveform analysis. PACE 1989; 12:1622-1630.

34. Chih-ming JC, Jenkins JM, DiCarlo LA. Digital signal processing chip implementation for detection and analysis of intracardiac electrograms. PACE 1994; 17:1373-1379.

35. Throne RD, Windle JR, Easley AR, et al. Scatter diagram analysis: A new technique for discriminating ventricular tachyarrhythmias. PACE 1994; 17:1267-1275. 
This document is a scanned copy of a printed document. No warranty is given about the accuracy of the copy. Users should refer to the original published version of the material. 